\title{
Effects of Plasma Volume and Red Cell Index on Cardiac Parameters for Shock Patients
}

Rabindra Nath Das ${ }^{1,2 *}$, Youngjo Lee ${ }^{2}$ and Gaurab Bhattacharyya ${ }^{1}$

${ }^{1}$ Department of Statistics, The University of Burdwan, Burdwan, West Bengal, India

${ }^{2}$ Department of Statistics, College of Natural Science, Seoul National University, Seoul, Korea

\begin{abstract}
Objectives: The report aims to identify the association of plasma volume and red cell index with some cardiac parameters based on 113 shock patients with 20 characters.
\end{abstract}

Background: In order to derive the association between plasma volume or red cell index with cardiac parameters, it is required to derive the model of any cardiac parameter as the response, while plasma volume, red cell index, along with other are explanatory variables. On the other hand, plasma volume (or red cell) index is the response, while cardiac parameters, along with others are explanatory variables. There is no such study in the literature.

Material and methods: The report is performed with 113 shock patients containing 20 characters, and the data set can be found in the site (http://www.umass.edu/statdata/statdata/data/shock.txt) Joint generalized linear statistical models have been applied.

Results: The mean plasma volume index (PVI) is negatively associated with diastolic blood pressure (DBP) $(P<0.0001)$, while it is positively associated with mean central venous pressure (MCVP) $(P<0.0001)$ and cardiac index $(\mathrm{Cl})(\mathrm{P}<0.0001)$. In addition, mean $\mathrm{PVI}$ is negatively associated with shock type $(\mathrm{SHOKT})$ at level $2(\mathrm{P}=0.0103)$. On the other hand variance of $\mathrm{PVI}$ is negatively associated with cardiac index $(\mathrm{Cl})(\mathrm{P}=0.0003)$, while the variance of DBP is positively associated with $\mathrm{PVI}(\mathrm{P}<0.0001)$. Again, mean red cell index $(\mathrm{RCl})$ is negatively associated with SHOKT at level $2(P=0.0522)$. The variance of $R C l$ is negatively associated with $\mathrm{Cl}(P=0.0397)$, while the variances of MCVP and HR are associated respectively, negatively with $R C I(P=0.0183)$, and positively with $R C l(P=0.0509)$.

Conclusion: The report shows $\mathrm{PVI}$ and $\mathrm{RCl}$ are strongly associated with either mean, or variance, or both of the considered cardiac parameters (SBP, DBP, MCVP, MAP, HR, Cl), which are given in the data set. The results in the report are completely new in the literature.

Keywords: Biochemical parameters; Cardiac parameters; Cardiac index; Mean arterial pressure; Plasma volume index; Red cell index; Joint generalized linear models (JGLMs)

\section{Introduction}

The red blood cells (RBCs) are known as erythrocytes. The RBCs normal size usually lies between 80 and $100 \mathrm{fL}$. Generally, blood red cell index (RCI) is a blood test which gives information regarding the hemoglobin content and red blood size mean corpuscular volume (MCV), where MCV denotes the average red blood cell size, which is computed by dividing the hematocrit (HCT) by the red cell count [1-3]. Many articles have suggested as potential predictors of cardiovascular disease (CVD) using, blood plasma volume, RCI associated with HCT and MCV, and white blood cells (WBC), along with its subtypes such as monocytes, lymphocytes and neutrophils [1,4-6]. On the other hand, blood plasma is the intravascular fluid which consists of dissolved proteins, water, glucose, electrolytes, clotting factors, carbon dioxide and hormones. All types of blood cells such as white blood cells, red blood cells, and platelets are suspended in plasma [7-9]. The average blood plasma of an individual is about $55 \%$ of the total body blood volume $[10,11]$. The blood plasma volume is about $40 \mathrm{~mL} / \mathrm{kg}$ of the body weight for female, whereas it is $39 \mathrm{~mL} / \mathrm{kg}$ for male [12]. A high blood plasma volume is associated with vitamin $\mathrm{C}$ deficiency, liver and spleen disease, while a low plasma volume is associated with dehydration, shock, and Addison's disease [13,14].

Recently, some articles have studied the determinants of some cardiac parameters such as SBP, DBP, MCVP, MAP, HR and CI for shock patients [15-19]. Many factors have been identified as the determinant of the above cardiac parameters. The following hypotheses have been tested in the report. Is there any association of PVI, or RCI with any one of the above cardiac parameters? If there is association, how are they associated with the cardiac parameters? What are the effects of PVI \& RCI on the above cardiac parameters? These hypotheses have been examined with the help of a real data set [20], and the data site is given in the Abstract.

\section{Materials}

A detail illustration of the considered data set in the report [20]. It contains 113 shock patients along with 20 explanatory characters. For ready reference, the characters are redisplayed as follows. The characters are: age (AGE), height (HEIGHT), sex (SEX) (male $=0$, female=1), SHOCKT (non-shock=1, hypovolemic=2, cardiogenic, or bacterial, or neurogenic or other=3), survival status (SURVIV) (survived=1, death=2), SBP, MAP, HR, DBP, MCVP, body surface index (BSI), CI, appearance time (AT), urinary output (UO), mean circulation time (MCT), PVI, hemoglobin (HG), hematocrit (HCT), $\mathrm{RCI}$, card record order (initial=1, final=2) $(\mathrm{CRO})$.

*Corresponding author: Rabindra Nath Das, Department of Statistics, The University of Burdwan, Burdwan, West Bengal, India, Tel: +91 3422634975 ; E-mail: rabin.bwn@gmail.com

Received September 24, 2018; Accepted September 29, 2018; Published September 30, 2018

Citation: Das RN, Lee Y, Bhattacharyya G (2018) Effects of Plasma Volume and Red Cell Index on Cardiac Parameters for Shock Patients. J Blood Lymph 8: 228. doi: $10.4172 / 2165-7831.1000228$

Copyright: (c) 2018 Das RN, et al. This is an open-access article distributed unde the terms of the Creative Commons Attribution License, which permits unrestricted use, distribution, and reproduction in any medium, provided the original author and source are credited. 


\section{Statistical Methods}

To examine the above stated hypotheses, appropriate probabilistic model to be developed for each cardiac parameter (treated as the response) on the remaining other characters (treated as the explanatory variables). On the other way, model to be prepared for PVI (or RCI) (treated as the response) on the remaining other characters. Some models of the cardiac parameters [15-19], and the models of PVI and $\mathrm{RCI}$ have been presented in the report. Note that all the interested responses (SBP, DBP, MCVP, MAP, HR, CI, PVI, RCI) are continuous, positive heteroscedatic, and belong to exponential family distribution. These are properly analyzed by JGLMs which is clearly given [21-24], and it is not reproduced explicitly in the report. To know more about JGLMs, interested readers are requested to go through the articles [21-24]. The responses PVI and RCI have been modeled using both the Log-normal and Gamma JGLMs, and it is found that Log-normal JGLMs give better fit for RCI, while Gamma JGLMs give better fit for PVI. Therefore, these two models are shortly reproduced herein.

\section{Log-normal JGLMs}

Considering a positive dependent continuous variable $y_{i}$ 's with $E\left(y_{i}\right)=\mu_{i}$ (mean parameters), and heteroscedastic variance $\sigma_{i}{ }^{2}$ (dispersion parameters), with $\operatorname{Var}\left(\mathrm{Y}_{\mathrm{i}}\right)=\sigma_{i}^{2} \mu_{\mathrm{i}}^{2}=\sigma_{i}^{2} V\left(\mu_{i}\right)$ say, the log transformation $\mathrm{Z}_{\mathrm{i}}=\log \left(\mathrm{Y}_{\mathrm{i}}\right)$ is frequently used to stabilize the variance $\operatorname{Var}\left(\mathrm{Z}_{\mathrm{i}}\right) \approx \sigma_{i}{ }^{2}$, but the variance is not stabilized always. For developing improved model, JGLMs for the mean and variance are applied. For Log-normal distributed positive random variable $\mathrm{Y}_{\mathrm{i}}$, with $\mathrm{Z}_{\mathrm{i}}=\log \mathrm{Y}_{\mathrm{i}}$, JGLM of the mean and variance are given by

$$
\begin{aligned}
& E\left(Z_{i}\right)=\mu_{z i} \text { and } \operatorname{Var}\left(Z_{i}\right)=\sigma_{z i}^{2}, \\
& \mu_{z i}=x_{i}^{t} \beta \text { and } \log \left(\sigma_{z i}^{2}\right)=g_{i}^{t} \gamma,
\end{aligned}
$$

Where $x_{i}^{t}$ and $g_{i}^{t}$ are the vectors of explanatory variables associated respectively, along with the regression coefficients $\beta$ (mean model parameters) and $\gamma$ (variance model parameters).

\section{Gamma JGLMs}

For a continuous positive random response $\mathrm{y}_{\mathrm{i},}$ if $E\left(y_{i}\right)=\mu_{i}$ and $\operatorname{Var}\left(y_{i}\right)=\sigma_{i}^{2} V\left(\mu_{i}\right)$, where $\mu_{\mathrm{i}}$ 's and $\sigma_{i}^{2}$ 's are respectively, mean and dispersion parameters, and $V\left({ }^{*}\right)$ reveals the variance function with two parts (in GLM) such as $\sigma_{i}^{2}$ (free of mean changes) and $V\left(\mu_{i}\right)$ (depends on the mean changes). It is noted that the GLM family distribution is located by $V\left(\mu_{i}\right)$, as it is Poisson if $V(\mu)=\mu$, Gamma if $V(\mu)=\mu^{2}$, and Normal if $V(\mu)=1$, etc. So, the Gamma JGLMs of mean and dispersion (when $V(\mu)=\mu^{2}$ ) are

$$
\eta_{i}=g\left(\mu_{i}\right)=x_{i}^{t} \beta \text { and } \varepsilon_{i}=h\left(\sigma_{i}^{2}\right)=w_{i}^{t} \gamma \text {, }
$$

where $g(\cdot)$ and $h(\cdot)$ are GLM link functions related to the mean and variance linear predictors respectively, and $w_{i}^{t}, w_{i}^{t}$ are the vectors of explanatory factors/variables, connected to the mean and dispersion parameters respectively. Practically, the maximum likelihood (ML) and the restricted ML (REML) method are used respectively, for estimating the mean and dispersion parameters [17].

\section{Association of PVI and RCI with cardiac parameters based on their analysis}

Analyses of SBP, DBP, HR, MCVP, MAP and CI for the given data set [15-19]. Based on these analyses we have the following conclusions.

- MCVP is positively associated with PVI ( $\mathrm{P}=0.0333)$, interpreting that MCVP increases as PVI increases [18].

- MCVP is positively partially associated with RCI ( $\mathrm{P}=0.3136)$, concluding that MCVP increases as RCI increases [18].

- Variance of MCVP (VMCVP) is positively partially associated with PVI $(\mathrm{P}=0.1228)$, indicating that VMCVP increases as PVI increases [18].

- VMCVP is negatively associated with RCI ( $\mathrm{P}=0.0183)$, implying that VMCVP increases as RCI decreases [18].

- Mean CI is positively associated with PVI $(\mathrm{P}<0.0001)$, interpreting that CI increases as PVI increases [19].

- MAP is negatively associated with RCI $(\mathrm{P}=0.0610)$, concluding that MAP increases as RCI decreases [16].

- Mean DBP is negatively associated with PVI ( $\mathrm{P}=0.0649)$, denoting that DBP increases as PVI decreases [15].

- Variance of DBP (VDBP) is positively associated with PVI $(\mathrm{P}<0.0001)$, indicating that VDBP increases as PVI increases [15].

- Variance of HR (VHR) is positively partially associated with PVI $(\mathrm{P}=0.0943)$, interpreting that VHR increases as PVI increases [17].

- VHR is positively associated with RCI $(\mathrm{P}=0.0509)$, concluding that VHR increases as RCI increases [17].

The above results are summarized in Table 1 . All the above conclusions are obtained from the analyses of the cardiac parameters (separately, each is treated as the response), and the remaining others are considered as the explanatory variables. Conversely, PVI (or $\mathrm{RCI}$ ) is treated as the response variable, and the remaining others are

\begin{tabular}{|c|c|c|c|c|c|c|}
\hline Model & Response Variable & Associated with & Estimate & S.E. & t-value & P-value \\
\hline Mean & MCVP & PVI & 0.0064 & 0.0030 & 2.142 & 0.0333 \\
\hline Mean & MCVP & $\mathrm{RCl}$ & 0.0020 & 0.0020 & 1.010 & 0.3136 \\
\hline Variance & MCVP & PVI & 0.0141 & 0.0091 & 1.549 & 0.1228 \\
\hline Variance & MCVP & $\mathrm{RCl}$ & -0.0340 & 0.0143 & -2.377 & 0.0183 \\
\hline Mean & $\mathrm{Cl}$ & PVI & 0.0117 & 0.0012 & 9.575 & $<0.0001$ \\
\hline Mean & MAP & $\mathrm{RCl}$ & -0.0008 & 0.0004 & -1.883 & 0.0610 \\
\hline Mean & DBP & PVI & -0.0009 & 0.0005 & -1.825 & 0.0694 \\
\hline Variance & DBP & PVI & 0.0360 & 0.0087 & 4.089 & $<0.0001$ \\
\hline Variance & HR & PVI & 0.0140 & 0.0085 & 1.680 & 0.0943 \\
\hline Variance & HR & $\mathrm{RCl}$ & 0.0420 & 0.0216 & 1.963 & 0.0509 \\
\hline
\end{tabular}
considered as the explanatory variables, the following analyses are derived.

Table 1: Association of Cardiac Parameters (SBB, MAP, HR, DBP, MCVP, Cl) with PVI and RCI. 
Citation: Das RN, Lee Y, Bhattacharyya G (2018) Effects of Plasma Volume and Red Cell Index on Cardiac Parameters for Shock Patients. J Blood Lymph 8: 228. doi: 10.4172/2165-7831.1000228

Page 3 of 6

\section{Analysis, results and interpretation of PVI}

Analysis of PVI: Plasma volume index (PVI) is treated as the response, and the remaining others are considered as the dependent variables, JGLMs analysis are performed under both Log-normal and Gamma models. The lowest value (in each class) of Akaike information criterion (AIC) accepts the final model by minimizing both the predicted additive errors and squared error loss [25]. It is noted that JGL Gamma models fit $(\mathrm{AIC}=1612.124)$ gives better results than Lognormal fit $(\mathrm{AIC}=1614)$. The derived results are shown in Table 2 . Some partially significant effects (known as confounder in epidemiology) are included in the model for better fitting [25]. In JGLMs, t-statistic is used to test the significance of each regression coefficient (Tables 2 and 3 ). For model diagnostic plots, the absolute residuals plot and normal probability plot are displayed in Figure 1.

Figure 1a shows the absolute residuals plot of Gamma fitted PVI models in Table 2, against the fitted values, which is almost a straight line, concluding that variance is constant. Figure $1 \mathrm{~b}$ (mean model of
PVI in Table 2) reveals the normal probability plot which does not show any discrepancy in fitting.

Results of PVI analysis: Table 2 shows that the mean PVI is negatively associated with the cardiac parameters DBP $(\mathrm{P}<0.0001)$ SHOCKT at level $2(\mathrm{P}=0.0103)$, and it is positively associated with MCVP $(\mathrm{P}<0.0001), \mathrm{CI}(\mathrm{P}<0.0001)$, while its variance is only negatively associated with $\mathrm{CI}(\mathrm{P}=0.0003)$. There are many more significant explanatory factors (in mean model) of PVI such as HEIGHT ( $\mathrm{P}=$ 0.0009), SEX $(\mathrm{P}<0.0001)$, SURVIV $(\mathrm{P}=0.0004)$, BSI $(\mathrm{P}<0.0001)$, CRO $(\mathrm{P}=0.0243)$, and also in variance model, other significant explanatory factor of PVI are AGE $(\mathrm{P}=0.0050)$ HEIGHT $(\mathrm{P}=0.0259)$, SEX $(\mathrm{P}=0.0223)$ and $\mathrm{UO}(\mathrm{P}=0.0303)$.

\section{Association of PVI with cardiac parameters based on PVI analysis}

From Table 2, the following associations of PVI with cardiac parameters can be noted.

\begin{tabular}{|c|c|c|c|c|c|}
\hline Model & Covariate & Estimate & s.e. & $t(214)$ & P-Value \\
\hline \multirow[t]{12}{*}{ Mean Model } & Constant & 4.4400 & 0.28998 & 15.311 & $<0.0001$ \\
\hline & HEIGHT & 0.0066 & 0.00197 & 3.346 & 0.0009 \\
\hline & SEX & -0.1944 & 0.02984 & -6.517 & $<0.0001$ \\
\hline & SURVIV & -0.1122 & 0.03120 & -3.595 & 0.0004 \\
\hline & SHOCKT (F2) & -0.0785 & 0.03035 & -2.586 & 0.0103 \\
\hline & SHOCKT (F3) & -0.0033 & 0.02865 & -0.115 & 0.9085 \\
\hline & DBP & -0.0033 & 0.00075 & -4.398 & $<0.0001$ \\
\hline & MCVP & 0.0117 & 0.00223 & 5.245 & $<0.0001$ \\
\hline & BSI & -0.7273 & 0.08838 & -8.229 & $<0.0001$ \\
\hline & $\mathrm{Cl}$ & 0.0452 & 0.00814 & 5.550 & $<0.0001$ \\
\hline & HCT & -0.0083 & 0.00195 & -4.260 & $<0.0001$ \\
\hline & CRO & 0.0520 & 0.02294 & 2.267 & 0.0243 \\
\hline \multirow[t]{7}{*}{ Dispersion Model } & Constant & 5.0798 & 2.7679 & 1.835 & 0.0675 \\
\hline & AGE & -0.0194 & 0.0068 & -2.833 & 0.0050 \\
\hline & HEIGHT & -0.0340 & 0.0152 & -2.243 & 0.0259 \\
\hline & SEX & -0.6254 & 0.2718 & -2.301 & 0.0223 \\
\hline & $\mathrm{Cl}$ & -0.2895 & 0.0798 & -3.629 & 0.0003 \\
\hline & UO & -0.0019 & 0.0009 & -2.180 & 0.0303 \\
\hline & HCT & -0.0209 & 0.0140 & -1.492 & 0.1371 \\
\hline
\end{tabular}

Table 2: Results for mean and dispersion models of PVI from Gamma fit.

\begin{tabular}{|c|c|c|c|c|c|}
\hline Model & Covariate & Estimate & s.e. & $t(218)$ & P-Value \\
\hline \multirow{8}{*}{ Mean Model } & Constant & 2.0914 & 0.20517 & 10.193 & $<0.0001$ \\
\hline & AGE & 0.0011 & 0.00092 & 1.186 & 0.2369 \\
\hline & SHOCKT (F2) & -0.0689 & 0.03532 & -1.952 & 0.0522 \\
\hline & SHOCKT (F3) & -0.0156 & 0.03974 & -0.394 & 0.6939 \\
\hline & $\mathrm{BSI}$ & -0.1877 & 0.08550 & -2.196 & 0.0291 \\
\hline & PVI & 0.0066 & 0.00114 & 5.746 & $<0.0001$ \\
\hline & HG & 0.0325 & 0.01254 & 2.588 & 0.0103 \\
\hline & $\mathrm{HCT}$ & 0.0148 & 0.00416 & 3.561 & 0.0004 \\
\hline \multirow{9}{*}{ Dispersion Model } & Constant & 3.7240 & 2.1193 & 1.757 & 0.0804 \\
\hline & HEIGHT & -0.0282 & 0.0126 & -2.240 & 0.0261 \\
\hline & SCHOKT (F2) & -0.1284 & 0.2966 & -0.433 & 0.6654 \\
\hline & SHOCKT (F3) & 0.5488 & 0.3101 & 1.769 & 0.0783 \\
\hline & SBP & 0.0051 & 0.0037 & 1.358 & 0.1758 \\
\hline & $\mathrm{Cl}$ & -0.1796 & 0.0868 & -2.069 & 0.0397 \\
\hline & UO & -0.0014 & 0.0009 & -1.560 & 0.1202 \\
\hline & PVI & -0.0140 & 0.0081 & -1.730 & 0.0850 \\
\hline & HCT & -0.0398 & 0.0149 & -2.661 & 0.0083 \\
\hline
\end{tabular}

Table 3: Results for mean and dispersion models of RCI from Log-normal fit. 


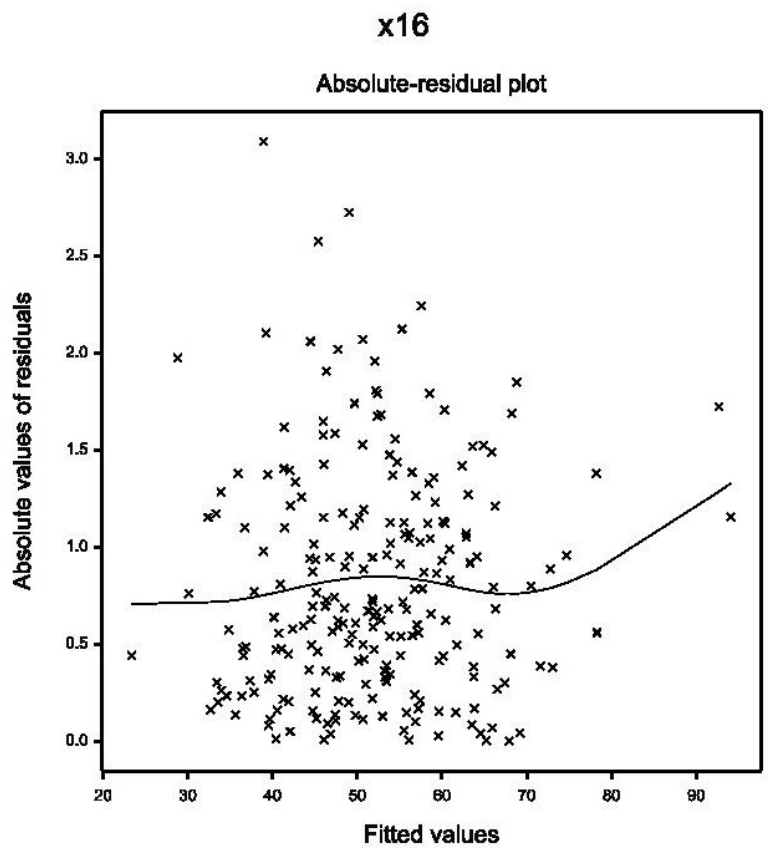

$\mathbf{a}$

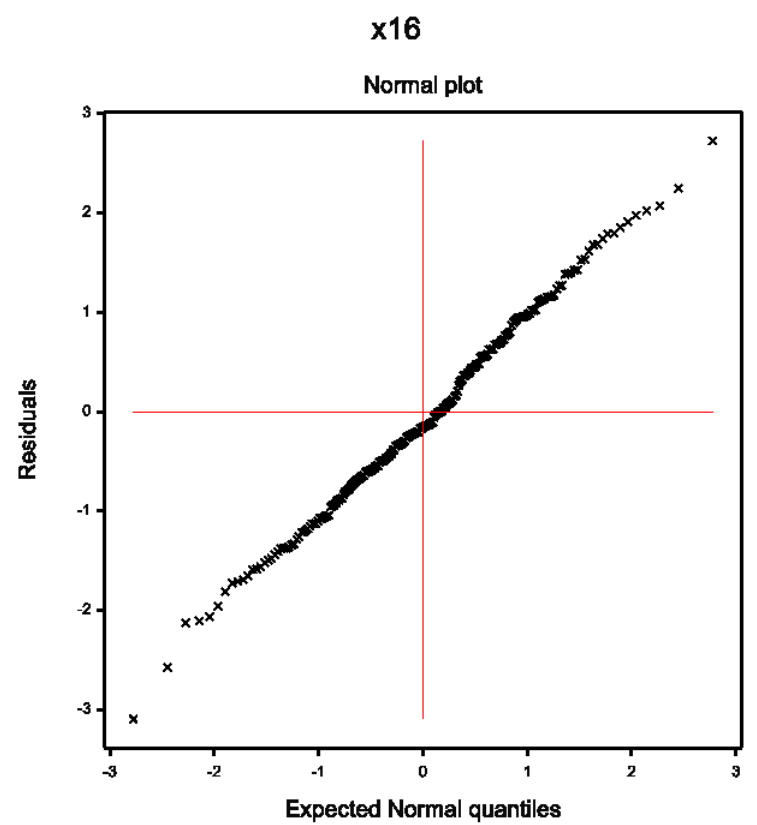

b

Figure 1: For the Gamma fitted models of PVI (Table 2) the (a) absolute residuals plot with respect fitted values, and (b) normal probability plot for the mean model.

- Mean PVI is negatively associated with DBP $(\mathrm{P}<0.0001)$, concluding that DBP increases as PVI decreases.

- Mean PVI is positively associated with MCVP $(\mathrm{P}<0.0001)$, interpreting that MCVP increases as PVI increases.

- Mean PVI is positively associated with CI $(\mathrm{P}<0.0001)$, concluding that $\mathrm{CI}$ increases as PVI increases.

- Mean PVI is negatively associated with SHOKT at level 2 $(\mathrm{P}=0.0103)$, concluding that as PVI decreases, incidence of shock will be increased at levels 2 than at level 1 .

- Variance of PVI is negatively associated with CI $(\mathrm{P}=0.0003)$, interpreting that PVI variance increases as $\mathrm{CI}$ increases.

All the above first three associations of PVI with the cardiac parameter are same as earlier results given in Table 1. The last two are new which are not derived in Table 1.

\section{Analysis, results and interpretation of RCI}

Analysis of RCI: Red cell index (RCI) is treated as the response, and the remaining others are considered as the dependent variables, JGLMs analysis is performed under both Log-normal and Gamma models. It is noted that JGL Log-normal models fit $(\mathrm{AIC}=1349)$ gives better results than Gamma fit $(\mathrm{AIC}=1370)$. The derived results are shown in Table 3. For model diagnostic plots, the absolute residuals plot and normal probability plot are displayed in Figure 2 .

Figure 2a shows the absolute residuals plot of Log-normal fitted RCI models in Table 3, against the fitted values, which is almost a straight line, concluding that variance is constant. Figure $2 \mathrm{~b}$ (mean model of RCI in Table 3) reveals the normal probability plot which does not show any discrepancy in fitting.
Results of RCI analysis: Table 3 shows that the mean RCI is negatively associated with the cardiac parameters SHOCKT at level $2(\mathrm{P}=0.0522)$ only, while its variance is negatively associated with SHOCKT at level $3(\mathrm{P}=0.0783)$ and $\mathrm{CI}(\mathrm{P}=0.0397)$, and it is positively partially associated with $\mathrm{SBP}(\mathrm{P}=0.1758)$. There are many more significant explanatory factors (in mean model) of RCI such as BSI $(\mathrm{P}=0.0291)$, PVI $(\mathrm{P}<0.0001)$, HG $(\mathrm{P}=0.0103)$, HCT $(\mathrm{P}=0.0004)$, and also in variance model, other significant explanatory factors of RCI are HEIGHT $(\mathrm{P}=0.0261)$, UO $(\mathrm{P}=0.1202)$, PVI $(\mathrm{P}=0.0850)$, and HCT $(\mathrm{P}=0.0083)$.

\section{Association of RCI with cardiac parameters based on RCI analysis}

From Table 3, the following associations of RCI with cardiac parameters can be noted.

- Mean RCI is negatively associated with SHOKT at level 2 ( $\mathrm{P}=0.0522)$, concluding that as RCI decreases, incidence of shock will be increased at levels 2 than at level 1 .

- Variance of RCI (VRCI) is negatively associated with CI $(\mathrm{P}=0.0397)$, interpreting that VRCI increases as $\mathrm{CI}$ decreases.

- $\quad$ VRCI is negatively partially associated with SHOKT at level 3 $(\mathrm{P}=0.0783)$, interpreting that $\mathrm{VRCI}$ increases at lower levels (1 \& 2) of SHOKT than at level 3.

- VRCI is positively partially associated with SBI ( $\mathrm{P}=0.1758)$, concluding that VRCI increases as SBI increases.

All the above associations of RCI with the cardiac parameter are completely new from Table 1 . 


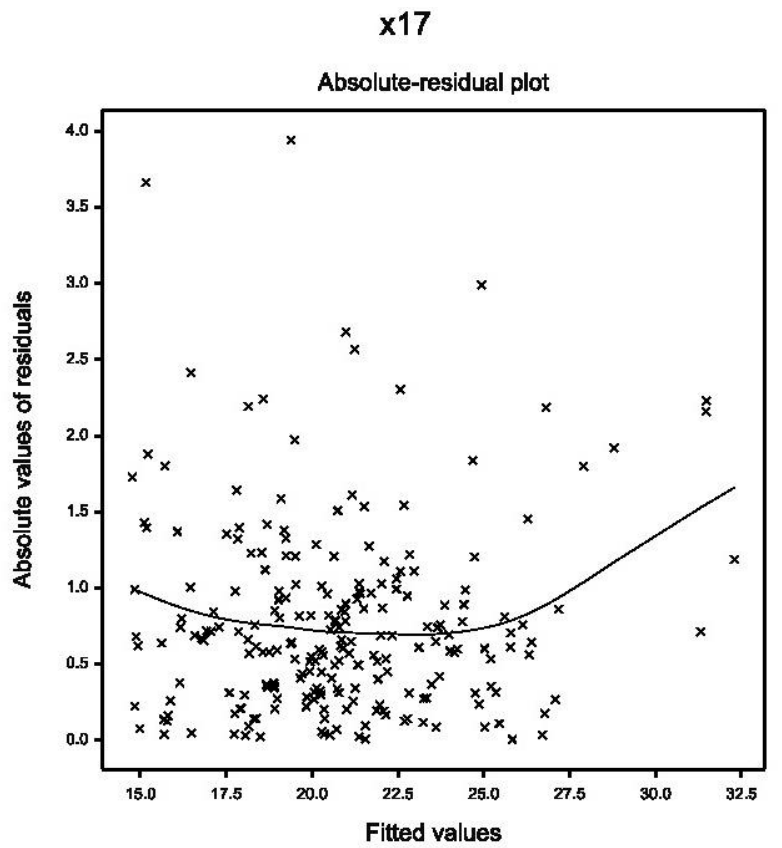

a

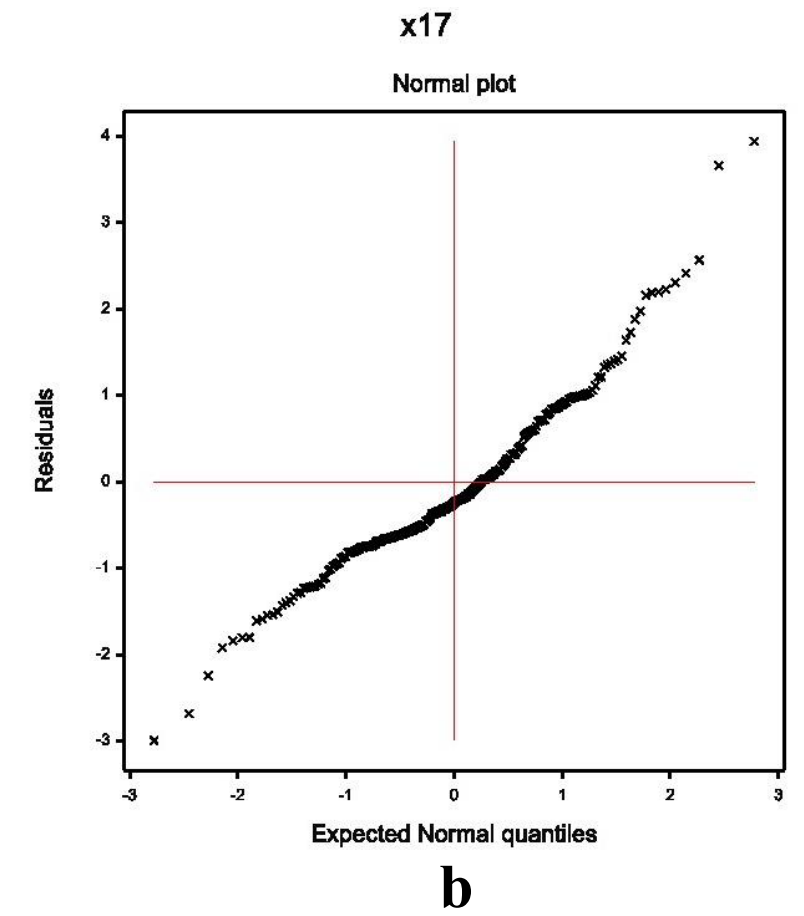

Figure 2: For the Log-normal fitted models of $\mathrm{RCl}$ (Table 3) the (a) absolute residuals plot with respect fitted values, and (b) normal probability plot for the mean model.

\section{Conclusion}

The report has focused the association of plasma volume and red cell index with the cardiac parameters (SBP, DBP, MCVP, MAP, HR, CI and Shock type) with the help of some probabilistic models that have derived by JGLMs. These associations have been established in two ways. The first way is, developing the joint mean and variance models of each cardiac parameter with PVI \& RCI, along with other explanatory characters. The second way is, developing the joint mean and variance models of PVI (or RCI) with the cardiac parameters, along with other characters. Model fitting has been examined by AIC, diagnostic plots, underlying distribution of the response variable, and along with the standard error of the estimates, which are very small (Tables 2 and 3), indicating that estimates are stable. In both ways, same associations have been established $[15,17]$.

The associations of PVI and RCI with cardiac parameters have been reported in the above three sections (Tables 1-3). It is shown that SBP is only partially associated with the variance of RCI (Table 3 ), whereas from mean models, MCVP and SHOCKT at level 2 are associated with both PVI \& RCI, mean CI, DBP are associated with PVI, and MAP is associated with RCI (Tables 1-3). Also variances of MCVP and HR are associated with both PVI \& RCI (Table 1), and DBP variance is associated with PVI (Table 1), whereas, CI is associated with the variances of PVI and RCI, and SHOCKT at level 3 is associated with the variance of RCI (Tables 2 and 3).

The above results and models are associated only with the consider data set [20]. For different data sets, models will be changed but the interpretation about the associations of PVI and RCI with the cardiac parameters may not be changed. It has not been verified for other data sets, as we have not different data. Moreover, we have not considered many other cardiac parameters such as basal BP, maximum BP, basal $\mathrm{HR}$, maximum $\mathrm{HR}$, peak $\mathrm{HR}$, ejection fraction etc., as these are not included in the data set. Future medical researchers may consider these covariates to examine the considered hypotheses herein.

From the above derived results, it can be concluded that MCVP will be high if PVI, or RCI, or both are high. CI will be high and DBP will be low if PVI is high. MAP will be low if RCI is high. Variance of MCVP will be high if PVI is high, or RCI is low. Variance of HR will be high if both PVI \& RCI are high. Variance of DBP will be high if PVI is high. Both variances of PVI \& RCI will be high if CI is low. From these derived associations, medical practitioners can predict the cardiac parameters based on the clinical report of PVI \& RCI. The causes of variation of the cardiac parameters such as SBP, DBP, HR, MCVP, MAP and CI may be explained with the values of PVI \& RCI.

\section{Conflict of Interest}

The authors confirm that this article content has no conflict of interest.

\section{Acknowledgement}

The authors are very much indebted to the referees who have provided valuable comments to improve this paper. This research was supported by the Brain Research Program through the National Research Foundation of Korea (NRF) funded by the Ministry of Science, ICT \& Future Planning (2014M3C7A1062896).

\section{References}

1. Arbel Y, Weitzman D, Raz R, Steinvil A, Zeltser D, et al. (2014) Red blood cell distribution width and the risk of cardiovascular morbidity and all-cause mortality: A population based study. Thromb Haemost 111: 300-307.

2. Puddu PE, Lanti M, Menotti A, Mancini M, Zanchetti A, et al. (2002) Red blood cell count in short-term prediction of cardiovascular disease incidence in the Gubbio population study. Acta Cardiol 57: 177-185.

3. Lippi G, Plebani M (2014) Red blood cell distribution width (RDW) and human pathology. One size fits all. Clin Chem Lab Med 52: 1247-1249.

4. Salvagno GL, Sanchis-Gomar F, Picanza A, Lippi G (2015) Red blood cell distribution width: A simple parameter with multiple clinical applications. Crit Rev Clin Lab Sci 52: 86-105. 
Citation: Das RN, Lee Y, Bhattacharyya G (2018) Effects of Plasma Volume and Red Cell Index on Cardiac Parameters for Shock Patients. J Blood Lymph 8: 228. doi: 10.4172/2165-7831.1000228

5. Mozos I (2015) Mechanisms Linking Red Blood Cell Disorders and Cardiovascular Diseases. BioMed Research International 2015: 1-12.

6. Libby $P(2002)$ Inflammation in atherosclerosis. Nature 420: 868-874.

7. Danesh J, Collins R, Appleby P, Peto R (1998) Association of fibrinogen, C-reactive protein, albumin, or leukocyte count with coronary heart disease: meta-analyses of prospective studies. JAMA 279: 1477-1482.

8. Matthew S, Welsch MA, Mitchell M, Vincent K, Braith RW, et al. (2000) Contracted Plasma and Blood Volume in Chronic Heart Failure. J Am Coll Cardiol 35: 51-55.

9. Wheeler JG, Mussolino ME, Gillum RF, Danesh J (2004) Associations between differential leucocyte count and incident coronary heart disease: 1764 incident cases from seven prospective studies of 30,374 individuals. Eur Heart J 25 1287-1292.

10. Waterhouse DF, Cahill RA, Sheehan F, McCreery CJ (2008) Prediction of calculated future cardiovascular disease by monocyte count in an asymptomatic population. Vasc Health Risk Manag 4: 177-187.

11. Duarte K, Monnez JM, Albuisson E, Pitt B, Zannad F, et al. (2015) Prognostic Value of Estimated Plasma Volume in Heart Failure. JACC Heart Failure 3: 886-893

12. Greenleaf JE, Convertino VA, Mangseth GR (1979) Plasma volume during stress testing in man: Osmolality and red cell volume. J Appl Physiol Respir Environ Exerc Physiol 47: 1031-1038.

13. Guasti L, Dentali F, Castiglioni L, Maroni L, Marino F, et al. (2011) Neutrophils and clinical outcomes in patients with acute coronary syndromes and/or cardiac revascularisation. Asystematic review on more than 34,000 subjects. Thromb Haemost 106: 591-599.
14. Das RN (2017) Systolic and Diastolic Blood Pressure Determinants of Shock Patients, J Heart Cardiol 3: 46- 52.

15. Das RN (2017) The mean arterial blood pressure determinants, EC Cardiology 4: $14-16$.

16. Das RN (2017) Shock patients heart rate variability factors. J Cardiovasc Med Ther 1: 19-23.

17. Das RN (2017) The Central Venous Pressure Causality Factors. J Gen Pract (Los Angel) 5: e116.

18. Das RN (2017) Cardiac Index Determinants. EC Cardiology 3: 112-114.

19. Afifi AA, Azen SP (1979) Statistical analysis: A computer oriented approach, 2nd ed. Academic Press, New York.

20. Lee Y, Nelder JA, Pawitan Y (2006) Generalized Linear Models with Random Effects (Unified Analysis via H-likelihood). London: Chapman \& Hall 2006.

21. Das RN, Lee $Y$ (2009) Log-normal versus gamma models for analyzing data from quality-improvement experiments. Quality Engineering 21: 79-87.

22. Das RN, Lee $Y$ (2010) Analysis strategies for multiple responses in quality improvement experiments. International Journal of Quality Engineering and Technology 1: 395-409.

23. Das RN (2014) Robust Response Surfaces, Regression, and Positive Data Analyses. London: Chapman \& Hall 2014.

24. Hastie T, Tibshirani R, Friedman J (2001) The Elements of Statistical Learning Springer-Verlag.

25. Das RN (2012) Discrepancy in fitting between log-normal and gamma models: An illustration. Model Assisted Statistics and Applications 7: 23-32. 